\title{
Measuring economic capital using loss distributions
}

\section{Osei Antwi", Alice Constance Mensah, Martin Owusu Amoamah, Dadzie Joseph}

Mathematics and Statistics Department, Accra Polytechnic, Accra-Ghana

Email address:

oseiantwi@yahoo.com (O.Antwi), alicemen_sah@yahoo.co.uk (A.C. Mensah)

\section{To cite this article:}

Osei Antwi, Alice Constance Mensah, Martin Owusu Amoamah, Dadzie Joseph. Measuring Economic Capital Using Loss Distributions. International Journal of Economics, Finance and Management Sciences. Vol. 1, No. 6, 2013, pp. 406-412.

doi: $10.11648 /$ j.ijefm.20130106.29

\begin{abstract}
This paper investigates the complexity involved in the quantitative measurement of Economic Capital and proposes simulation methods as a practical solution for obtaining the loss distribution of a portfolio of obligors. The paper examines a one factor model to generate loss distribution which establishes the necessary ingredients to measure the credit risk quantities in a loan portfolio. The general elements of credit risk modeling are outlined and then a specific model that employs a Monte Carlo simulation is developed. An example is provided that calculates the risk quantities in a loan portfolio from which the Economic Capital in a credit risk portfolio is obtained.
\end{abstract}

Keywords: Economic Capital, Unexpected Loss, Obligor, Asset Value Correlation, Joint Probability of Default

\section{Introduction}

Economic Capital (EC), the amount of capital required to absorb unexpected losses in the coming year at a portfolio's manager's level of confidence, is the risk benchmark for organisations that have to manage portfolios of credit-risk assets of loans and bonds. EC usually gives a measure of the amount required to cover a bank's losses with a certain probability or confidence level. Economic capital analysis typically involves an identification of the risks from certain activities or exposures, an attempt to measure and quantify those risks, and an attribution or allocation of capital to those risks. The EC has now increasingly become an accepted input into decision-making at various levels within banking organisations.

Despite the growing popularity of EC there are still a lot of people and organisations that find it hard to understand the economic capital concept and compute it. Many people are intimidated by the mathematics that underlies the EC models, and there seems to be a lack of literature that bridges the gap between daily credit risk management and complex capital modeling.

The concept of Economic Capital dictates that banks should hold some capital as cushion against unexpected losses. Some banks may use internally developed models to calculate their ECs. However, banks may also use commercial software to assist them in their EC calculations. A typical example of such software for credit risk is the Portfolio Manager by Moody's KMV, Strategic Analytics, Credit risk + by Credit Suisse and CreditMetrics by JP
Morgan. Value-at-risk (VaR) models are also typical EC frameworks for market and other risks.

Currently, Basel II accord proposes Economic Capital for AAA banks in the region of $99.9 \%$. We shall employ a Monte Carlo simulation procedure to produce a loss distribution capable of determining the EC at such confidence levels. This will make it possible for financial institutions using the default approach to determine precisely the amount of capital needed to cushion them against future unexpected losses and improve greatly the bank's capital adequacy requirements as stipulated in BASEL II.

Our approach of determining EC will be based on a single-risk factor calculation methodology that allows easy analytical solutions, rather than full-blown multi-factor models. At its essence, the model will generate a distribution of portfolio credit losses, which can be decomposed into Expected Loss (EL) and Unexpected Loss (UL) components. Conceptually, EL is akin to the mean loss rate of a portfolio and represents the loss that a bank can reasonably anticipate will occur over a given time horizon. EL is simply an aggregated measure of the PD of each obligor in the portfolio multiplied by its LGD, since the bank can expect to lose an amount equivalent to the likelihood of each obligor defaulting adjusted by the proportion of the exposure that will not be recovered upon that default. Capital charges are then designed to cover Unexpected Losses (UL). However, defining the UL of a portfolio as the risk capital saved for cases of financial distress is not the best choice, because there might be a 
significant likelihood that losses will exceed the portfolio's expected loss by more than one standard deviation of the portfolio loss. Therefore one seeks other ways to quantify risk capital, hereby taking a target level of statistical confidence into account.

The determination of EC using the proposed methodology allows the quantification of risk capital at various confidence levels.

For a prescribed level of confidence $\alpha$ the EC is defined as the $\alpha$ - quantile of the portfolio loss $L_{P F}$ minus the $E L$ of the portfolio,

$$
E C_{\alpha}=q_{\alpha}-L_{P F}
$$

where $q_{\alpha}$ is the $\alpha$-quantile of $L_{P F}$. For example, if the level of confidence is set to $\alpha=99.98 \%$, then the risk capital $E C_{\alpha}$ will (on average) be sufficient to cover unexpected losses in 9,998 out of 10,000 years, hereby assuming a planning horizon of one year. The reason for reducing the quantile $q$ by the $E L$ is due to the decomposition of the total risk capital (i.e., the quantile) into a first part covering expected losses and a second part meant as a cushion against unexpected losses.

In effect, all risk quantities of the credit portfolio and therefore the economic capital can be identified by means of the loss distribution of the portfolio. This is an important observation, because it shows that in cases where the distribution of the portfolio loss can only be determined in an empirical way one can use empirical statistical quantities as a proxy for the respective "true" risk quantities.

In practice, there are essentially two ways to generate a loss distribution. The first method is based on Monte Carlo simulation; the second is based on a so-called analytical approximation. In a Monte Carlo simulation, losses are simulated and tabulated in form of a histogram in order to obtain an empirical loss distribution of the underlying portfolio. Approaching the loss distribution of a large portfolio by Monte Carlo simulation always requires a sound factor model. The classical statistical reason for the existence of factor models is the wish to explain the variance of a variable in terms of underlying factors.

Despite the fact that in credit risk we also wish to explain the variability of a firm's economic success in terms of global underlying influences, the necessity for factor models comes from two major reasons. First of all, the correlation between single loss variables should be made interpretable in terms of economic variables, such that large losses can be explained in a sound manner. For example, a large portfolio loss might be due to the downturn of an industry common to many counterparties in the portfolio. Along this line, a factor model can also be used as a tool for scenario analysis. For example, by setting an industry factor to a particular fixed value and then starting the Monte Carlo simulation again, one can study the impact of a down or upturn of the respective industry.

Often, one-factor models admit a decomposition of the portfolio loss variable into a monotonic function of the factor and a residual. The former part of the decomposition is called systematic risk whereas the latter part is called specific or idiosyncratic risk. Kalkbrener (2007) showed that the Deutsche Bank uses factor models in the determination of its EC measures. The IRB approach of Basel II regulatory framework is also based on credit risk modeling concepts that are broadly consistent with credit economic capital models used increasingly by financial institutions to measure portfolio-level risk and to manage and allocate capital across the enterprise.

Sometimes, the portfolio loss variable converges in some sense to a monotonic function of the factor. This observation can be used as point of departure for analytic approximations of important statistics of the portfolio like quantiles of the portfolio loss variable (value-at-risk, VaR). Gordy (2003) was the first to suggest this approach which he called granularity adjustment for the one-factor model credit portfolio that underlies the Basel II regulatory capital rules which came into force in 2007 (see BCBS, 2004). Martin and Wilde (2002) observed that the results by Gouri'eroux et al. (2000) make feasible an easier and more systematic way to derive the adjustments. The purpose of the granularity adjustment is to recognise that a bank with exposures characterised by coarse granularity, implying a large residual of undiversified idiosyncratic risk (i.e. singleborrower risk concentrations), should require additional capital. Similarly, a bank with exposures characterised by finer than typical or average granularity should demand a smaller than average capital requirement.

The second reason for the need of factor models is a reduction of the computational effort. For example, for a portfolio of 100,000 transactions, $2 \times 100,000 \times 99,000$ correlations have to be calculated. In contrast, modeling the correlations in the portfolio by means of a factor model with 100 indices reduces the number of involved correlations by a factor of $1,000,000$.

The other approach to the determination of portfolio loss distribution is by analytical approximation method. Roughly speaking, the analytical approximation maps an actual portfolio with unknown loss distribution to an equivalent portfolio with known loss distribution. The loss distribution of the equivalent portfolio is then taken as a substitute for the "true" loss distribution of the original portfolio. We shall concentrate on the determination of loss distribution using the first approach, which is by Monte Carlo simulation.

The study will thus;

- $\quad$ Establish the frame work to compute loss distribution for a portfolio of obligors

- Compute the Economic Capital from the loss distribution of a portfolio of obligors

- Obtain the risk quantities such as Expected Loss and Unexpected Loss of the portfolio

\section{Economic Capital Modeling}

We shall begin to construct the Economic Capital framework by developing the structures that constitute the economic capital. Before going into the details of this construction we shall first look at factor models. 


\subsection{Credit Risk Models}

In last few years, several models of credit risk have been developed. The four main types of industry models can be classified into Asset Value Models, Macroeconomic Models, Actuarial Models and Intensity Models. We shall basically focus on factor models, a form of Asset Value Models (AVMs), more specifically, the one factor model.

Factor models are well established multivariate statistics, applied in credit risk models for identifying drivers of correlated defaults and for reducing the computational effort regarding the calculation of correlated losses. The asset value process models developed mainly by Moody's KMV and Credit Metrics focus on default-only mode. These models are of Bernoulli type deciding about default or survival of a firm by comparing the firm's asset value at a certain horizon with some critical threshold. Factor models falls into this class of asset value process models. In factor models, it is assumed that the asset value process is dependent on some underlying factors reflecting industrial and regional influences, thereby driving the economic future of the firm. Factor model focuses on the asset value returns $r_{i}$ of the counterparties $(i=1,2,3, \ldots \ldots . m)$ at the planning horizon admitting a representation

$$
r_{i}=\beta_{i} \Phi_{i}+\varepsilon_{i}
$$

Where $\Phi_{i}$ is called the composite factor of firm $i$. The variable $\varepsilon_{i}$ represents the residual part of $r_{i}$. Essentially $\mathcal{E}_{i}$ is the error one makes in substituting $r_{i}$ by $\beta_{i} \Phi_{i}$.

\subsection{The One Factor Model}

The one factor model assumes that a single factor common to all counterparties influences the economic fortunes of the counterparties and that the asset correlation between obligors is uniform. We shall look at a model consisting of a portfolio of individual assets which are influenced mainly by a single factor - the state of the economy.

The one factor model is completely described by specializing Equation (1) to the case of only one single factor common to all counterparties hereby assuming that the asset correlation between obligors is uniform. More explicitly, this means that the composite factor $\Phi$ of all obligors is equal to one single factor, usually denoted by $Y \sim N(0,1)$. Hence, Equation (1) is substituted by

$$
r=\sqrt{\rho} Y+\sqrt{(1-\rho)} Z_{i}, \quad i=(1 \ldots \ldots \ldots . \quad m)
$$

where $\sqrt{1-\rho} Z_{i}$, with $Z \sim N(0,1)$, takes over the role of residual $\varepsilon_{i}$ and $\rho$ is the uniform asset correlation between the asset value log-returns $r \sim N(0,1)$. In one factor models, it is assumed that the residuals $Z_{i}$ constitute an independent family, also independent of the factor $Y$.

\subsection{Unexpected Loss}

The standard deviation of Expected Loss measures the credit risk of loan transaction. The standard deviation of Expected Loss is typically called the Unexpected Loss abbreviated by UL.

\subsubsection{Unexpected Loss of a Single Credit Risk Transaction}

The unexpected Loss of a credit risk transaction is a function of the PD, LGD, and EAD and their variances. PD, LGD and EAD are independent random variables.

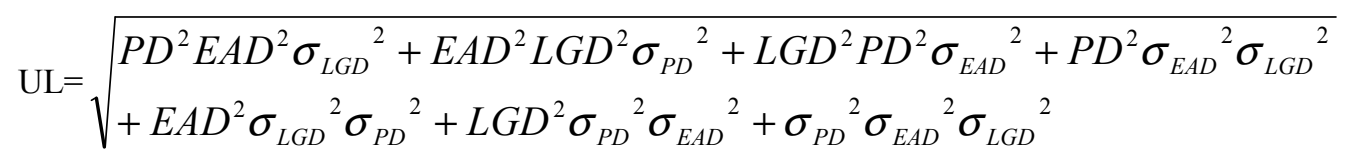

All the functions are independent and we will assume that the probability of default has a Bernoulli distribution, so that we can substitute $\sigma_{P D}^{2}$ by $\left(P D-P D^{2}\right)$.

The Loss Given Default has a Beta distribution, which allows us to replace $\sigma_{L G D}^{2}$ by $L G D \times \frac{(1-L G D)}{4}$, and

EAD is assumed to be deterministic, so that $\sigma_{E A D}^{2}=0$. This leads to the equation:

$$
\text { Unexpected Loss }=E A D \times \sqrt{\left[L G D^{2} \times P D \times(1-P D)+P D \times L G D \times(1-L G D) / 4\right.}
$$

\subsubsection{Unexpected Loss of a Portfolio}

Due to diversification (we can spread our investment over various positions in different industry sectors and regions), the unexpected loss of the portfolio is given by: 


$$
\text { Unexpected Loss of a Portfolio }=\sqrt{\sum_{i=1, \ldots, n} \sum_{i=1, \ldots, n} U L_{i} \times U L_{j} \times \rho_{i, j}},
$$

Where $\sigma_{i, j}$ represents the default correlation between obligor $i$ and obligor $j$ in a portfolio of $n$ obligors. For a portfolio of two obligors where $n=2$, the unexpected

$$
\text { Portfolio Unexpected Loss }=U L_{P L}=\sqrt{U L_{1}^{2}+U L_{2}{ }^{2}+2 U L_{1} U L_{2} \sigma_{1,2}}
$$

where $\sigma_{1,2}$ is the default correlation between obligor 1 and obligor 2. The default correlation gives an indication of the tendency of the two loans to default at the same point in time. We are now left to determine the default correlation between obligor 1 and obligor 2, i.e. finding $\sigma_{1,2}$. We shall proceed from here to determine the general default correlation $\sigma_{i, j}$ between obligor $i$ and obligor $j$.

\subsection{Default Correlation of Two Loans}

The default correlation gives an indication of the tendency of the two loans to default at the same point in time. The default correlation between two obligors is a function of their $P D_{i}$ and the $J P D_{j}$. The default correlation is determined by the equation:

$$
\sigma_{i, j}=\frac{J P D-P D_{i} \times P D_{j}}{\sqrt{P D_{i} \times\left(1-P D_{i}\right)\left(1-P D_{j}\right)}}
$$

where $J D P$ is the joint probability of default of obligor $i$ and obligor $j . P D_{i}$ and $P D_{j}$ are the probability of default of obligor $i$ and obligor $j$. To determine the default correlation of the two obligors we first need to calculate the Asset Value Correlation (AVC) of the two obligors and then use this information to derive the Joint Default Probability of the two obligors which will allow us to determine the default correlation.

\subsection{The Asset Value Correlation (AVC)}

The asset value correlation of the obligors is obtained by: Asset return obligor 1

$$
r_{1}=\left(\sqrt{R^{2}{ }_{1}}\right) * Y+\left(\sqrt{1-R^{2}{ }_{1}}\right) * \varepsilon_{1}
$$

The Asset return of obligor 2 is a function of the systematic factor and a specific factor to obligor 2 .

Asset return obligor 2

$$
r_{2}=\left(\sqrt{R_{2}^{2}}\right) * Y+\left(\sqrt{1-R_{2}^{2}}\right) * \varepsilon_{2}
$$

From Equations 8 and 9, it is clear that the common factor that drives the changes in the asset values of both obligors is $\mathrm{Y}$ (the composite factor), which represents the change in the state of the economy. Thus, the asset correlation between obligor 1 and obligor 2 is solely influenced by the systematic factor common to both obligor 1 and obligor 2 .

\subsection{The Joint Probability of Default (JPD)}

The Joint Probability of Default is the default thresholds of the obligors behaviour which are functions of the value of each obligor's liabilities. If the asset value of an obligor falls below the obligor's default threshold, the obligor defaults. The Joint Probability of Default is a function of the PD's of the obligors and their asset correlation.

$$
J D P=J D P\left(P D_{i}, P D_{j} \text {, asset correlatio } n_{i, j}\right)
$$

The Joint Probability of Default is established by determining the volume under the asset value distribution up to the default threshold of the two obligors. Mathematically, the JPD is a double integral which we approximate by a Visual Basic program in Excel.

\subsection{Economic Capital Requirements of a Loan Portfolio}

The Unexpected Loss as determined by Equation 5 represents credit risk at two standard deviation of loss. The portfolio unexpected loss thus provides confidence level of around $90 \%$. This falls short of the confidence levels that are used in current practice to determine economic capital, which typically ranges between $99.5 \%$ and $99.98 \%$. Suppose we use a confidence level of $99 \%$, this implies that our tolerance for actual losses exceeding our economic capital is $1 \%$. It is important to note that though we have suggested $99 \%$ to illustrate the economic capital concept, in practice, institutions usually calculate economic capital using confidence level that reflects their desired credit rating.

\section{Graph of a Typical Loss Distribution Curve}

Figure 1 shows the levels of Expected Loss, Unexpected Loss and the Economic Capital in a graph of a typical loss distribution. 


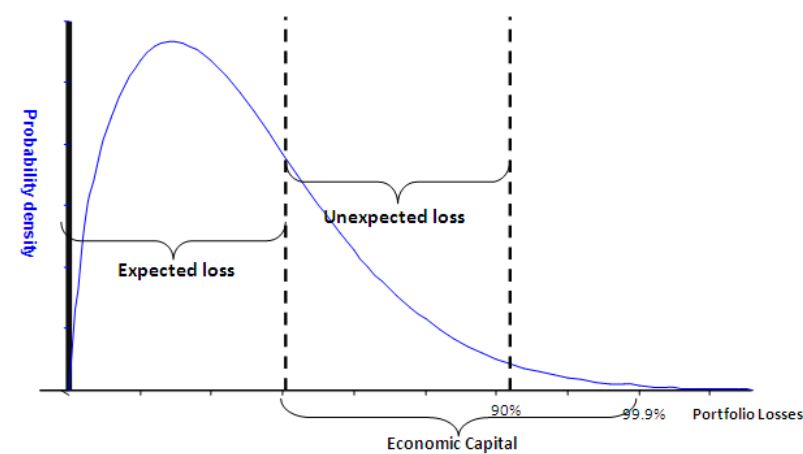

Figure 1. Loss Distribution Curve

\section{Monte Carlo Simulations}

Due to the shortcomings of the Unexpected Loss in determining how much capital to hold against future loses, we determine the EC by using Monte Carlo simulation. The main advantage of using Monte Carlo simulation is that it accurately captures the correlations inherent in the portfolio instead of relying on a whole bunch of assumptions. Moreover, Monte Carlo simulation takes into account all the different risk characteristics of the loans in the portfolio. In contrast to closed-form solutions that involve solving theoretical formulas and equations, Monte Carlo simulations is an alternative way to obtain the aggregate loss distribution in which an algorithm is cleverly implemented in a computer and it does the job.

The Monte Carlo Simulation of credit risk using the one factor can be split into five main steps:

1. Specify the Probabilities of Default (PDs) of the portfolio.

2. Specify LGD which is given by

$100 \times(1-$ Recovery Rate)

3. Specify EAD, the Exposure at Default Unexpected loss of the portfolio is given by $\sum(E A D \times P D \times L G D)$

4. Specify the asset value correlations of individual credit events.

5. Based on steps 1-4, obtain the portfolio loss via Monte Carlo simulations.

Probability of default can be obtained using any of these three approaches:

- Structural models

- Logit scores

- Historical default rates per rating category

The LGDs can be obtained by using historical average of LGDs or multivariate prediction model. In the asset value approach, we simulate asset values by generating random values and finding the inverse. The random values generated $(0,1)$ are not normally distributed but the inverse (inverseRand( )) is standard normally distributed. If the asset values are normally distributed then

$$
\text { Default Point }=\Phi^{-1}(P D)
$$

where $\Phi$ denotes the cumulative standard normal distribution function.

Let $d_{i}$ denote the default point such that if the borrower's asset value falls below the default point (which is a function of the borrower's liability), the borrower defaults, otherwise borrower survives.

$$
\begin{gathered}
\text { Default; } \Leftrightarrow A_{i} \leq d_{i} \\
\text { No Default; } \Leftrightarrow A_{i} .>d_{i}
\end{gathered}
$$

We now run the Monte Carlo simulation for a portfolio of hundred (100) obligors. We perform the simulations using the steps $1-4$.

For one scenario, we set $\mathrm{EAD}$ at $£ 10,000,000,000$. If we assume a recovery rate of $40 \%$ then, $\mathrm{LGD}=60 \%$. PD is set at $20 \%$ given a Default Point $=-0.842$. The default correlation is also set to $\rho=0.51$. It is possible to vary the PD and $\rho$. In such a case, a different scenario is generated. This procedure can be repeated to generate infinite number of scenarios. We now perform the simulations using the procedure. The results are summarised in the loss frequency table in Appendix A. The loss distribution is as shown in Figure 2.

Contents of Appendix A can now be summarised thus:

- The columns $1 \& 2$ from the left-hand-side shows the specifications of EAD, PD, Default point, LGD and $\rho$. Also, Expected Loss and total number of simulations performed.

- Column 3 from the Left-Hand-Side shows the Cumulative Portfolio Losses which are in multiples of $£ 6,000,000$ (LGD is $60 \%$ so if EAD is $£ 10,000,000$, then each default will lead to the loss of $60 \% \mathrm{x}$ $£ 10,000,000=£ 6,000,000)$.

- Column 4 shows the Frequency or Probability of the losses.

- Column 5 shows the portfolio loss under each scenario of the macroeconomic condition or a given macro factor.

- Column 6 shows the simulated macro factor (which is the state of the economy at each point in time).

The remaining columns show the obligors situation under the default condition:

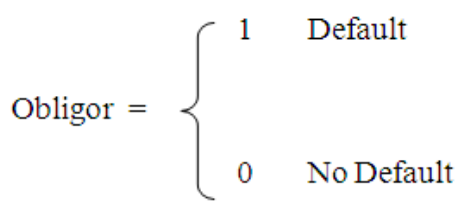




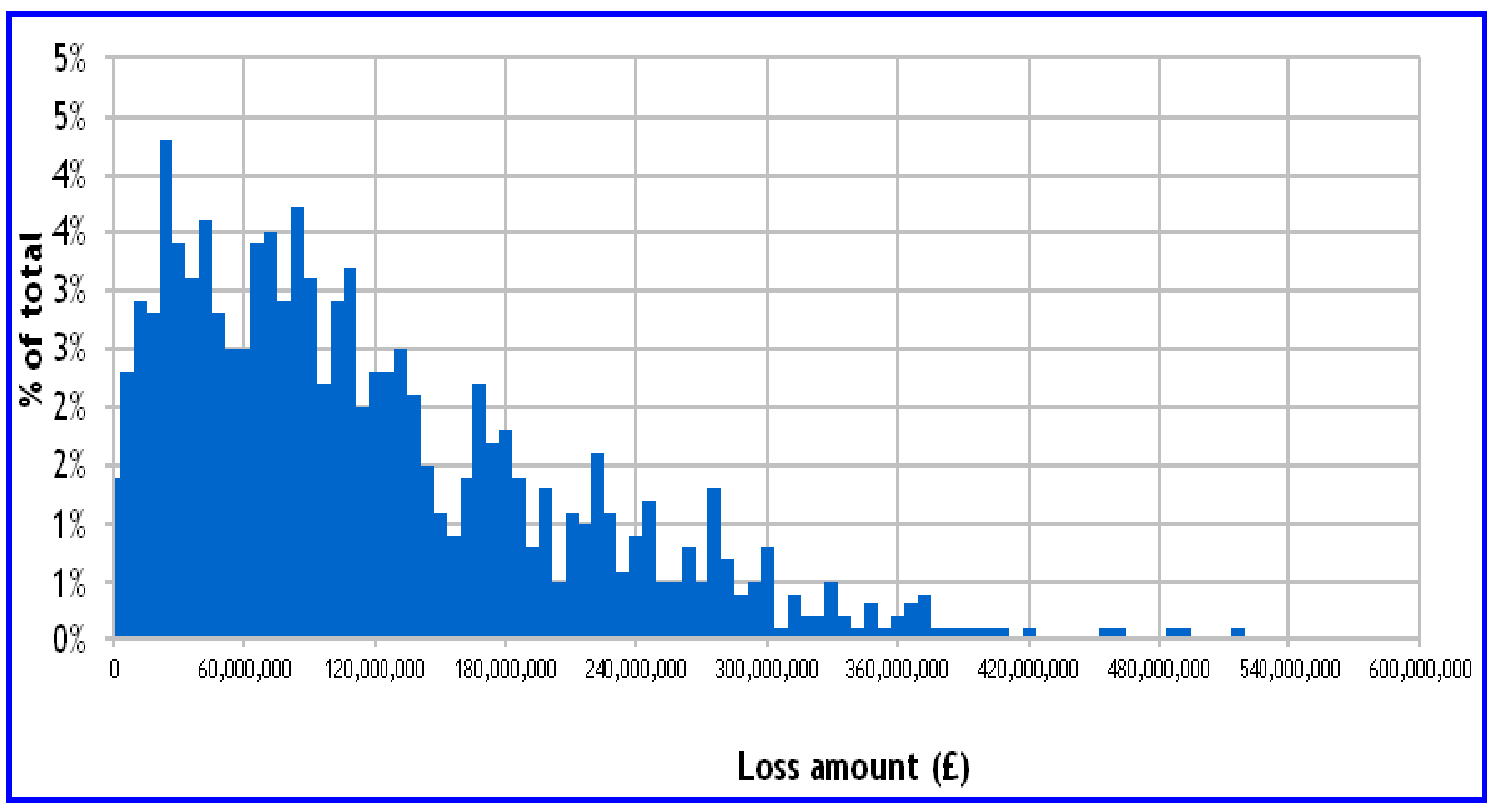

Figure 2. Loss Distribution from Monte Carlo Simulation

Depending on a lending institution's rating or risk appetite, it world set the confidence interval for its EC. We give here the EC required for three institutions whose EC covers up to $95 \%, 99 \%$ and $99.9 \%$ confidence interval.

Table 2. Ranking Table of Loss Amount

\begin{tabular}{cccc}
\hline $\begin{array}{c}\text { Confidence } \\
\text { Interval }\end{array}$ & & Rank & Loss Amount \\
\hline $95.0 \%$ & $5.0 \%$ of $1000=50$ & Top 50 & $£ 282,000,000$ \\
$99.0 \%$ & $1.0 \%$ of $1000=10$ & Top 10 & $£ 414,000,000$ \\
$99.9 \%$ & $0.1 \%$ of $1000=1$ & Top 1 & $£ 450,000,000$ \\
\hline
\end{tabular}

Table 3. Capital Requirements at Different Confidence Levels

\begin{tabular}{cccc}
\hline $\begin{array}{c}\text { Confidence } \\
\text { Level }\end{array}$ & Value at Risk & $\begin{array}{c}\text { Expected } \\
\text { Loss }\end{array}$ & $\begin{array}{c}\text { Economic } \\
\text { Capital }\end{array}$ \\
\hline $95.0 \%$ & $£ 282,000,000$ & $£ 120,000,000$ & $£ 162,000,000$ \\
$99.0 \%$ & $£ 414,000,000$ & $£ 120,000,000$ & $£ 294,000,000$ \\
$99.5 \%$ & $£ 450,000,000$ & $£ 120,000,000$ & $£ 330,000,00$ \\
\hline
\end{tabular}

For example, for a $95 \%$ confidence level, the amount of loss corresponding to the $95 \%$ confidence interval is $5 \%$ of 1,000 (loss scenarios generated $=1,000$ ) when ordered from largest to lowest. So we aggregate 50 losses from the top of the loss. That is the $50^{\text {th }}$ largest loss when ordered from largest to lowest. Table 2 provides the computations and Table 3 provides the (rank) or losses at different confidence levels of $95 \%, 99 \%$ and $99.9 \%$.

\section{Conclusions}

As we have seen, loss simulation results are simple to obtain and interpret. Percentiles at different confidence levels are also possible to obtain using this approach. In addition, the methods also provide estimates up to 99.9 percentile which is what is required by institutions with high rating. This level of accuracy is usually difficult to obtain by traditional methods. Thus, the simulation approach advanced here provide us with is a very convenient tool to generate aggregate loss distribution and obtain all the risk quantities in a credit portfolio which is of critical importance for risk managers and regulators alike. We believe the model we have proposed here would solve the Economic Capital problem and help banks and lending institutions in their daily risk management. The model does not require extensive mathematical rigor. In fact, one needs only to understand how to obtain the Monte Carlo simulations in order to obtain the loss distributions. 
Appendix A. Table of Values of Loss Frequency

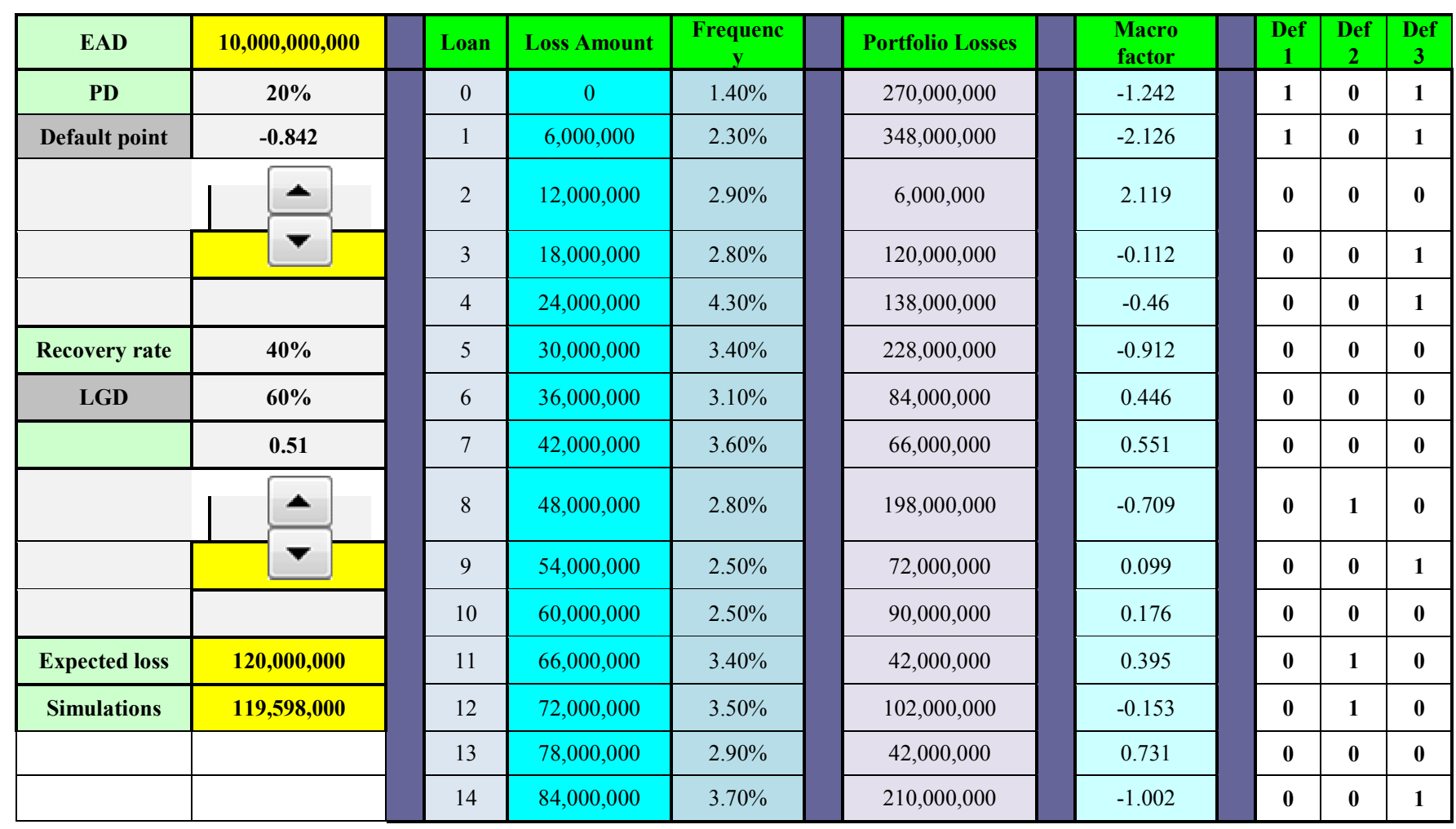

\section{References}

[1] Bluhm, Ludger Overbeck., Wagner, Christoph., An Introduction to Credit Risk Modeling, Christian Chapman \& Hall/CRC Financial Mathematics Series, pages 15-121,

[2] Hoogbruin, Peter-Paul., Journal of Global Association of Risk Professionals, September/October 2006 issue, pages 34-39.

[3] Haigh, John., Probability Models, Springer Undergraduate Mathematical Series, Springer, pages 1-86

[4] Barreto, Humberto., Howland, Frank M., Introductory Econometrics, Cambridge University Press, 2006, pages 215-235.

[5] David Vose, Risk Analysis A Quantitative Guide, John Wiley \& Sons Ltd., 2003, page 59

[6] Berenson, Mark L., Levine, David M., Basic Business Statistics, Prentice-Hall International inc., Seventh Edition 1999, pages 291-330
[7] Winston, Wayne; Financial Models Using Simulation and Optimization II. Palisade Corporation NY. 2001, pages 9-20

[8] Addison-Wesley, Visual Basic an Object Oriented Approach, Pearson Education Ltd., 2001, pages 1-202

[9] Enrique Navarrete, Practical Calculation of Expected and Unexpected Losses on Operational Risk by Simulation Methods.

[10] Michael Kalkbrener (Deutsche Bank's Economic Capital Model LRC - Risk Analytics \& Instruments, COMISEF Tutorial on Quantitative Finance, London, 18 October 2007.

[11] Martin, Hansen., Dr. Gary, van Vuuren., Mariarosa, Verde., Basel II Correlation Values., An Empirical Analysis of EL, UL and the IRB Model, Credit Market Research Financial Institutions Special Report, Fitch Rating 2008, Page 3.

[12] Bank for International Settlements, The New Basel Capital Accord, Consultative Document Basel Committee on Banking Supervision May 2001, Page 84. 